\title{
ANÁLISIS DE SUELO Y FOLIAR EN GUANÁBANO (Annona muricata L.) EN BLANCA ESPUMA MUNICIPIO DE ALTO LUCERO, VERACRUZ ${ }^{1}$
}

\author{
LIBRADO VIDAL HERNÁNDEZ², GABRIELA SÁNCHEZ VIVEROS ${ }^{2}$ \\ NAYELLI AYATZOL VIDAL MARTÍNEZ², DORIS GUADALUPE CASTILLO ROCHA², \\ ROBERTO GREGORIO CHIQUITO CONTRERAS ${ }^{2}$
}

RESUMEN-El presente estudio se realizó en una plantación de guanábano de siete años de edad. Se efectuaron análisis de suelo y foliar para determinar las características físicas y químicas del suelo y el estado nutrimental del árbol, durante dos épocas climáticas (seca y lluvia). En cada una de ellas se obtuvieron ocho muestras de suelo a una profundidad de 0 a $30 \mathrm{~cm}$, las cuales se mezclaron para obtener dos muestras compuestas para su análisis. La recolecta foliar se realizó en un árbol productivo y en otro no productivo, de la parte media del árbol y se consideró la cuarta hoja de la rama. El objetivo fue generar información sobre valores referenciales preliminares sobre los aspectos nutricionales del guanábano en un agroecosistema comercial y en condiciones del trópico subhúmedo. La textura del suelo analizado fue clasificada como de migajón-arcillosa. El contenido de la materia orgánica fue alto y el $\mathrm{pH}$ fue moderadamente ácido para ambas épocas. Durante la estación de seca los valores nutrimentales químicos del suelo encontrado en relación a los macroelementos y microelementos fueron altos y adecuados, en tanto que en el período de lluvia, estos fueron variables de alto, deficiente y adecuado. Los valores en el análisis foliar en la concentración de los macroelementos y microelementos en ambas épocas fueron adecuados.

Termos para indexacion: Contenido nutrimental, $\mathrm{pH}$, macroelementos, microelementos.

\section{SOIL AND FOLIAR IN SOURSOP (Annona muricata L.) IN BLANCA ESPUMA TOWNSHIP OF ALTO LUCERO, VERACRUZ}

\begin{abstract}
This study was conducted in a seven years old plantation. Soil and foliar analyzes were performed to determine the physical and chemical characteristics of the soil and the nutrient status of the tree for two climatic seasons (dry and wet). In each of these eight samples were obtained from soil at a depth of 0 to $30 \mathrm{~cm}$, which were mixed to obtain two compound samples for analysis. The leaf gathering was performed in a production tree and other non-productive, from the middle part of the shaft and the fourth leaf of the branch was considered. The objective was to generate information on preliminary reference values on nutritional aspects of a commercial agroecosystem soursop and humid tropical conditions. Analized soil texture was classified as loamy-clayey. The organic matter content was high and moderately acid $\mathrm{pH}$ for both periods. During the dry season soil chemical nutrient values related to macro-and microelements were high and adequate, while in the rainy season, they were high, poor and adequate. The values in the leaf analysis in the concentration of macro-and microelements in both seasons were adequate.
\end{abstract}

Index terms: Nutrient content, $\mathrm{pH}$, macroelements, microelements.

${ }^{1}$ Palestra Anonáceas - V Congresso Internacional \& Encontro Brasileiro sobre Annonaceae: do gene à exportação (19 a 23 de agosto de 2013). Botucatu-SP.

${ }^{2}$ Académicos de la Facultad de Ciencias Agrícolas. Universidad Veracruzana Campus Xalapa. Circuito Gonzalo Aguirre Beltrán S/N . Zona Universitaria. C.P.91190. Xalapa Veracruz, México.E-mails: 1vidal152@hotmail.com; gabrielauv@gmail.com; ayatzol.vidal@ gmail.com; dorisgcr@gmail.com y contrerasch@yahoo.com 


\section{INTRODUCCIÓN}

En México existen muchas regiones agroecológicas que cumplen con las condiciones necesarias para el desarrollo del guanábano. La superficie cultivada es de 7,250 ha de este frutal, con un volumen estimado de $54375 \mathrm{~T}$. Se ha determinado que la productividad está definida por diversos factores, destacando el medio ambiente, la planta, el suelo y la tecnología de producción empleada (VIDAL, 2006).

La nutrición del guanábano es uno de los factores más importante para aumentar la productividad y al mismo tiempo mejorar la calidad de los frutos. Sin embargo, por diversos motivos, en la mayor parte de las zonas productoras de guanábano del país esta práctica no se realiza. Las tierras donde se cultiva el guanábano son muy diferentes, varían de región a región, incluso de parcela a parcela. Existe diferencia por clase de tierra, edad de la planta, época del año y sistema de producción. Esto lógicamente plantea un problema, pero se resuelve conociendo cuáles son los requerimientos nutricionales del guanábano, principalmente después de cada cosecha.

La nutrición de los frutales es compleja, la detección de limitaciones nutrimentales que afectan al rendimiento entre una amplia cantidad de otros factores es difícil. Factores ambientales o biológicos son, frecuentemente limitantes y la respuesta en rendimiento no siempre se observa aún cuando se haya corregido un problema nutrimental. No es entonces sorprendente que la respuesta en rendimiento de ensayos de campo sea muchas veces inconsistente. Definir el estado nutrimental óptimo es, por lo tanto, un proceso a largo plazo, caro y difícil (VERGARA, 1990).

El diagnóstico del estado nutrimental de los árboles puede ser afectado por factores como la fecha de muestreo, tipo de hoja muestreada, etapa fenológica del cultivo, uso de valores de referencia incorrectos, entre otros (MALDONADO y VERGARA, 1993).

Existen pocos trabajos de investigación sobre la nutrición del guanábano. Avilán (1975), en Venezuela, encontró que las plantas de guanábano durante su desarrollo varían la demanda de los elementos en orden de importancia tenemos, potasio, nitrógeno, calcio, fósforo y magnesio. Además, plantea los niveles adecuados y deficientes de estos elementos a nivel foliar: Nitrógeno (N) 1.10\% (deficiente); 1.76\% (adecuado): Fósforo (P) 0.11\% (deficiente); $0.29 \%$ (adecuado): Potasio (K) $1.26 \%$ (deficiente); $2.60 \%$ (adecuado): Calcio (Ca) 1.08\% (deficiente); $1.76 \%$ (adecuado) y Magnesio $(\mathrm{Mg})$
$0.68 \%$ (deficiente); $0.20 \%$ (adecuado).

Castro (1996) reporta que en la región de Palmira, Colombia los valores adecuados de los macroelementos y microelementos en hojas de guanábano son: N: $1.75 \%$; P: $0.29 \%$; K: $2.6 \%$; Ca: $1.76 \%$; Mg: 0.20\%; S: 0.2-0.3\%; Fe: $60-120$ mg kg-1; Mn: 24-200 mg kg-1 B: 35-100 mg kg-1; $\mathrm{Cu}: 5-12 \mathrm{mg} \mathrm{kg}^{-1}$ y Zn: $20-25 \mathrm{mg} \mathrm{kg}^{-1}$. En tanto los valores deficientes fueron: $\mathrm{N}: 1.10 \%$; P: $0.11 \%$; K: 1.26\%; Ca: $1.08 \%$; Mg: 0.08\%; S: 0.14\%; Fe: $35 \mathrm{mg}$ $\mathrm{kg}^{-1}$; Mn: $16 \mathrm{mg} \mathrm{kg}^{-1}$; B: $20 \mathrm{mg} \mathrm{kg}^{-1}$; Cu: $4 \mathrm{mg} \mathrm{kg}^{-1}$ y Zn: $16 \mathrm{mg} \mathrm{kg}^{-1}$. Sin embargo, el autor no señala las características del árbol, fenología, época de muestreo y tipo de manejo.

En México se localizaron dos estudios sobre guanábano realizados en el estado de Tabasco. Álvarez (1996) reporta valores nutrimentales de $\mathrm{N}$ : 2.27\%; P: 0.30\%; K: 2.03\%; Ca: 1.86\%; Mg: 0.28\%; Na: $0.06 \mathrm{mg} \mathrm{kg}^{-1}$; Fe: $219 \mathrm{mg} \mathrm{kg}^{-1}$; Mn: $68 \mathrm{mg} \mathrm{kg}^{-1}$; $\mathrm{Cu}: 29 \mathrm{mg} \mathrm{kg}^{-1}$ y Zn: $32 \mathrm{mg} \mathrm{kg}^{-1}$. El autor señala que son adecuados, es decir, el cultivo está bien abastecido por el suelo. Al igual que Castro (1996) no hace comentario sobre las condiciones que prevalecieron durante el desarrollo de la investigación. González (2003) reporta que el muestreo foliar lo realizó en tres épocas del año, época de seca, lluvia y norte y en cuatro agrosistemas. Los valores registrados durante la época de seca y el agrosistema Las Lilias fueron N: 1.80\%; P: $0.20 \%$; K: 1.26\%; Ca: 3.08\%; Mg: 0.17\%; Na: $0.3 \mathrm{mg} \mathrm{kg}^{-1}$; S: $24.8 \mathrm{mg} \mathrm{kg}^{-1}$; Fe: $87 \mathrm{mg} \mathrm{kg}^{-1}$; Mn: $1 \mathrm{mg} \mathrm{kg}^{-1}$; Cu: $12 \mathrm{mg} \mathrm{kg}^{-1}$ y Zn: $20.3 \mathrm{mg} \mathrm{kg}^{-1}$. Mientras que los valores reportados durante la época de lluvia fueron N: 1.76\%; P: 0.15\%; K: 1.24\%; Ca: 1.34\%; Mg: 0.16\%; Na: $0.43 \mathrm{mg} \mathrm{kg}^{-1}$; S: $155.11 \mathrm{mg}$ $\mathrm{kg}^{-1}$; Fe: $154 \mathrm{mg} \mathrm{kg}^{-1}$; Mn: $8 \mathrm{mg} \mathrm{kg}^{-1}$; Cu: $6 \mathrm{mg} \mathrm{kg}^{-1} \mathrm{y}$ $\mathrm{Zn}: 18 \mathrm{mg} \mathrm{kg}^{-1}$. El autor no hace comentario sobre las condiciones que prevalecieron durante el desarrollo de la investigación.

En Veracruz, el cultivo del guanábano, ha generado un interés creciente entre los diferentes productores agrícolas de la región, debido a los precios y la demanda nacional e internacional que ha llegado a adquirir esta fruta. Para el cultivo del guanábano se cuenta con un área costera del Golfo de los 0 a $800 \mathrm{msnm}$ siendo una superficie de 30,460 ha con muy buen potencial productivo, desde Tuxpan hasta las Choapas, Veracruz.

El guanábano representa una alternativa de producción en el estado, ya que además de contar con condiciones agroecológicas adecuadas para su cultivo, se dispone de selecciones con caracteres de alto rendimiento, resistentes y/o moderadamente resistentes al barrenador de la semilla, antracnosis y bajas temperaturas. Asimismo se cuenta con 
portainjertos apropiados para su propagación asexual, aprovechando las características propias de estos. En Veracruz, aunque son pocos los productores de guanábano, hay muchos interesados en iniciarse en este cultivo, pero la falta de información tecnológica sobre la nutrición y la problemática fitosanitaria bajo las condiciones de suelo y agroecología de nuestro estado, ha limitado el desarrollo del cultivo. Por lo que el objetivo del presente trabajo fue generar información sobre valores referenciales preliminares sobre los aspectos nutricionales del guanábano en un agroecosistema comercial y en condiciones del trópico subhúmedo.

\section{MATERIALES Y MÉTODOS}

El presente trabajo de investigación se realizó en una plantación comercial de guanábano, ubicado en la localidad de Blanca Espuma, municipio de Alto Lucero, Ver., ubicado entre las coordenadas $19^{\circ} 34^{\prime}$ 47" Latitud Norte y las 96 42'06" Longitud Oeste. Se encuentra localizado a una altitud de $619 \mathrm{~m}$. Es una plantación de siete años de edad, 2.5 ha con árboles de pie franco, con un sistema de plantación rectangular 6 x 5 y 3.5 ha con árboles injertos distribuidos en un sistema de marco real $5 \times 5$. Existía un programa de control de plagas y enfermedades, manejo de malezas, fertilización y riego.

Dentro de la plantación se realizó análisis de perfil de suelo en donde se hicieron dos excavaciones para su estudio.

El área de estudio fue dividido en cuatro cuadrantes en base a la pendiente suelo. En cada cuadrante se tomaron cuatro muestras, considerándose una repetición. Los muestreos se realizaron en zigzag. Y las barrenaciones de suelo a una profundidades $0-30 \mathrm{~cm}$ en las dos épocas climáticas (seca y lluvia) en cada uno de los cuadrantes. Se colectó para cada cuadrante cuatro muestras. Las cuatros muestras se mezclaran y se seleccionó una cuarta parte de esta mezcla, considerándose a esta última la muestra ideal. Por cada muestreo por época climática se tuvieron dos muestras, haciendo un total de 4 muestras de suelos analizados. El muestreo foliar se realizó de acuerdo a la metodología usada por Elizondo (1989), se colectó la cuarta hoja a partir del meristemo, por cada árbol, ubicadas en el estrato medio distribuidas hacia los cuatro cuadrantes del árbol. El muestreo se realizó en un árbol productivo y otro no productivo. El diseño experimental utilizado fue completamente al azar con dos tratamientos y cuatro repeticiones.

El análisis de suelo y foliar se realizaron en el Laboratorio de Fertilidad y Nutrición Vegetal del
Campo Experimental de Cotaxtla, perteneciente al INIFAP. A fin de determinar el nivel nutrimental, las variables evaluadas fueron: Textura, Materia Orgánica (MO), pH, N, Fósforo (P), Potasio $(\mathrm{K})$, Calcio (Ca), Magnesio $(\mathrm{Mg})$, Sodio $(\mathrm{Na})$, Hierro (Fe), Cobre (Cu), Zinc (Zn), Manganeso $(\mathrm{Mn})$, Azufre (S) y el análisis foliar consistió en determinar las concentraciones de macroelementos y microelementos.

\section{RESULTADOS Y DISCUSIÓN}

En relación a las características físicas del suelo durante la época de seca (marzo) el análisis de perfil de suelo, el horizonte A mostró un color negro y una textura arcillosa, con cierta pedregosidad. El horizonte B un color gris y textura sumamente arcillosa (barro), éstas no son apropiadas para el guanábano, sin embargo, como el manejo del cultivo se encuentra en condiciones de riego y fertilización, su sistema radical es superficial, sin necesidad de profundizar en el suelo. La textura del suelo en base a los resultados del análisis de las muestras recolectadas en la época de seca y de lluvia fueron: Arena: $52.20 \%$; Arcilla: $26.80 \%$ y Limo: $21.00 \%$ según la NOM 021 SEMARNAT 2000 es un suelo con textura migajón arcilloso. El contenido de la materia orgánica (MO) fue de 3.68\% según la NOM 021 SEMARNAT 2000, el contenido es alto. El pH fue de 6.32 según la NOM 021 SEMARNAT 2000, es moderadamente ácido. En cuanto a las características físicas del suelo son adecuadas para el crecimiento y desarrollo del guanábano (VIDAL, 1993).

El contenido de los macroelementos durante la época seca fue de N: 2.09\%; P: 1.35\%; K: 18.08\%; Ca: $51.46 \%$ y Mg: $57.00 \%$ valores altos según la NOM 021 SEMARNAT 2000, 11 y 12 (Figura 1). Sin embargo, se observó en los árboles de guanábano un crecimiento y producción normal.

El contenido de los microelementos durante la época seca fue de Fe: $17.75 \mathrm{mg} \mathrm{kg}^{-1}$; Cu: $1.40 \mathrm{mg}$ $\mathrm{kg}^{-1}$; Zn: $2.10 \mathrm{mg} \mathrm{kg}^{-1}$ y Mn: $19.7 \mathrm{mg} \mathrm{kg}^{-1}$ adecuado según NOM 021 SEMARNAT 2000 (Figura 2). El comportamiento de los árboles en cuanto a crecimiento vegetativo, floración y fructificación fue normal, sin embargo, la incidencia de plagas y enfermedades estuvo presente en un $40 \%$.

El análisis de la variable concentración de los macroelementos foliar para el árbol productivo fue de N: $1.74 \%$; P: $0.02 \%$; K: 1.59\%; Ca: $2.62 \%$ y Mg: $0.20 \%$ (Figura 3). Estos valores los reporta Avilán (1975) como deficientes. Sin embargo, Castro (1996) y González (2003) como adecuados.

En el árbol no productivo la concentración 
de N: $2.53 \%$; P: $0.04 \%$; K: 3.05\%; Ca: $0.98 \%$ y Mg: $0.15 \%$ los valores son más alto en relación con el árbol productivo, tal vez esto se deba a que no sean aprovechados durante la floración amarre y crecimiento del fruto (Figura 4). Esto coincide con lo reportado por Avilán (1975) que los considera normales para el guanábano.

En relación a la variable análisis foliar en la concentración de los microelementos en el árbol productivo presentó valores deficientes $\mathrm{Cu}: 2.6 \mathrm{mg}$ $\mathrm{kg}^{-1} \mathrm{y}$ altos en Fe: $149 \mathrm{mg} \mathrm{kg}^{-1}$; Mn: $57.5 \mathrm{mg} \mathrm{kg}^{-1}$ adecuados y altos en Zinc: $381 \mathrm{mg} \mathrm{kg}^{-1}$, en tanto que en árboles no productivos el análisis reporta valores deficientes en $\mathrm{Cu}: 7.1 \mathrm{mg} \mathrm{kg}^{-1}$ así como en Mn: 9.3 mg kg-1 y altos en Fe: $135 \mathrm{mg} \mathrm{kg}^{-1}$ y Zn: $481 \mathrm{mg}$ $\mathrm{kg}^{-1}$ (Castro, 1996), (Figuras 5 y 6).

Los resultados obtenidos del análisis de las muestras de suelo durante la época de lluvia no variaron las propiedades físicas del suelo. Sin embargo, fueron muy variables las propiedades químicas del suelo. El contenido de nitrógeno fue de $1.54 \%$ siendo un valor mediano según la NOM 021 SEMARNAT 2000. En tanto que fósforo fue de 0.48\% siendo un contenido bajo según la NOM 021 SEMARNAT 2000. Por otro lado, K: 6.53\%; Ca: 17.48\% y Mg: $4.87 \%$ fueron valores altos según NOM 021 SEMARNAT 2000 (Figura 7).

Los contenido de los microelementos en la muestra No. 1 los contenido de Fe: $1.95 \mathrm{mg} \mathrm{kg}^{-1}$ fue deficiente según la NOM 021 SEMARNAT 2000. En tanto que $\mathrm{Cu}: 0.80 \mathrm{mg} \mathrm{kg}^{-1} \mathrm{Zn}: 1.6 \mathrm{mg} \mathrm{kg}^{-1}$ y Mn: $1.4 \mathrm{mg} \mathrm{kg}^{-1}$ fueron adecuados según la NOM 021 SEMARNAT 2000 y el B: $1.10 \mathrm{mg} \mathrm{kg}^{-1}$ el contenido fue mediano según la NOM 021 SEMARNAT 2000. En la muestra No. 2 el Fe: $2.1 \mathrm{mg} \mathrm{kg}^{-1} ; \mathrm{Zn}: 0.74 \mathrm{mg}$ $\mathrm{kg}^{-1}$ y B: $0.28 \mathrm{mg} \mathrm{kg}^{-1}$ el contenido de estos elementos fueron deficientes según NOM 021 SEMARNAT 2000 y 15 ; mientras que $\mathrm{Cu}: 0.70 \mathrm{mg} \mathrm{kg}^{-1}$ y $\mathrm{Mn}$ : $1.40 \mathrm{mg} \mathrm{kg}^{-1}$ fueron adecuados según la NOM 021 SEMARNAT 2000 (Figura 8).

En el análisis foliar la concentración de los macroelementos en el árbol productivo para $\mathrm{N}$ : 1.96\%; P: 0.11\%; K: 2.74\%; Mg: $0.22 \%$ y Ca: $1.80 \%$. Estos valores coinciden con lo reportado por Castro (1996) que los considera adecuados. Con relación al árbol no productivo los valores fueron $\mathrm{N}: 1.82 \%$; P: $0.10 \%$; K: 2.05\%; Mg: 0.19\% y Ca: 3.41\%. Éste último presentó valores más altos (Figura 9 y 10).

En relación a la concentración de los microelementos el árbol productivo presentó valores de Fe: $158 \mathrm{mg} \mathrm{kg}^{-1}$; Cu: $4.5 \mathrm{mg} \mathrm{kg}^{-1}$; Zn: $530 \mathrm{mg} \mathrm{kg}^{-1}$; Mn: $8 \mathrm{mg} \mathrm{kg}^{-1}$ y B: $39 \mathrm{mg} \mathrm{kg}^{-1}$. Para Castro (1996) los valores de $\mathrm{Fe}$, y $\mathrm{Zn}$ son altos y para $\mathrm{Cu}$ y $\mathrm{Mn}$ son deficientes. En tanto que en el árbol no productivo los valores más altos fueron en $\mathrm{Na}: 359 \mathrm{mg} \mathrm{kg}^{-1}$ (Figuras 11 y 12).

\section{Contenido de macroelementos (\%)}

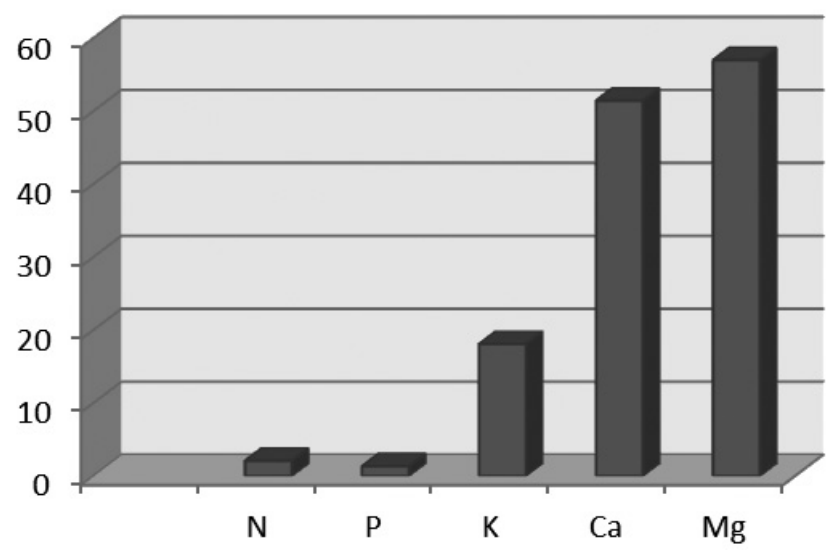

Época de seca

FIGURA 1- Contenidos de los macroelementos con valores altos en Ca y $\mathrm{Mg}$. 


\section{Contenido de microelementos $\left(\mathrm{mg} \mathrm{kg}^{-1}\right)$}

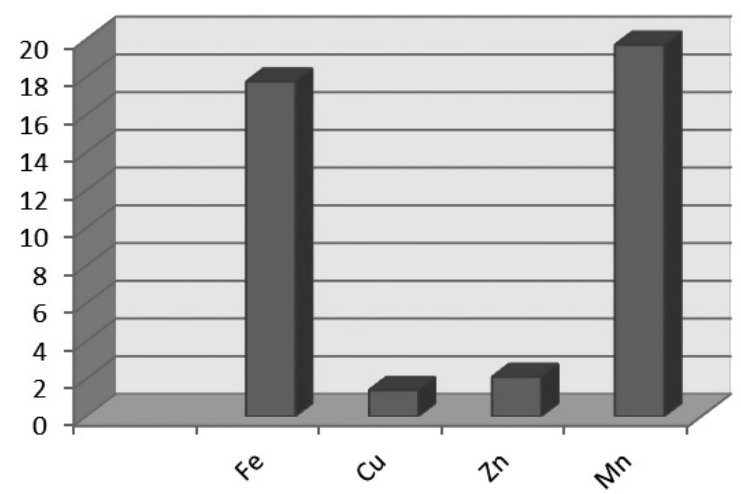

$\square$ Época de seca

FIGURA 2- Contenidos de los microelementos con valores altos en Fe y Mn.

\section{Árbol productivo}

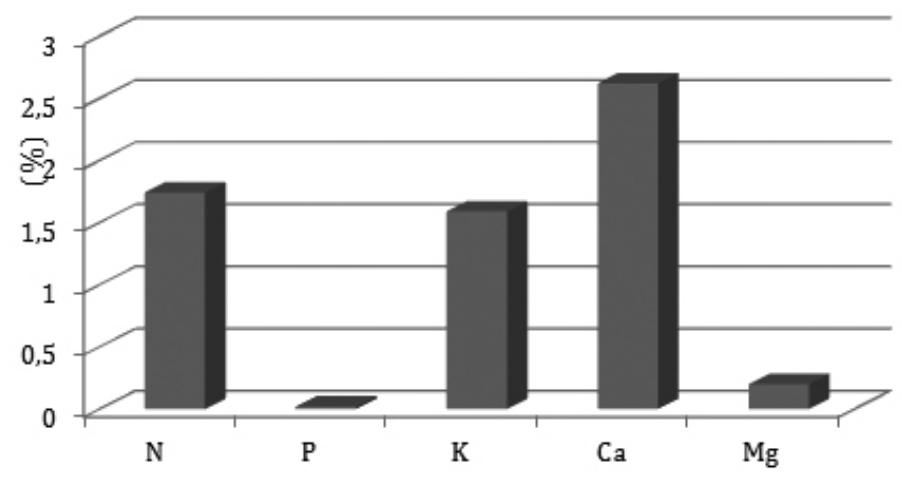

FIGURA 3- Concentración de los macroelementos con valor alto de Ca.

\section{Árbol no productivo}

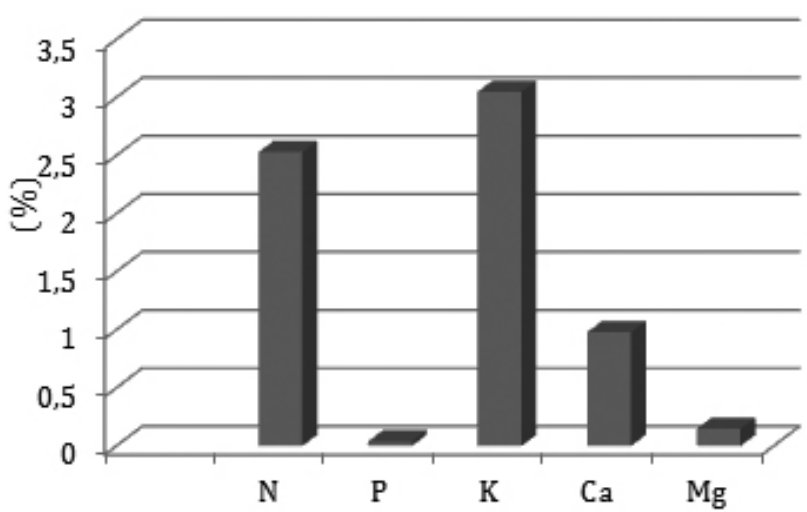

FIGURA 4- Concentración de los macroelementos en el análisis foliar durante la época seca. 


\section{Árbol productivo}

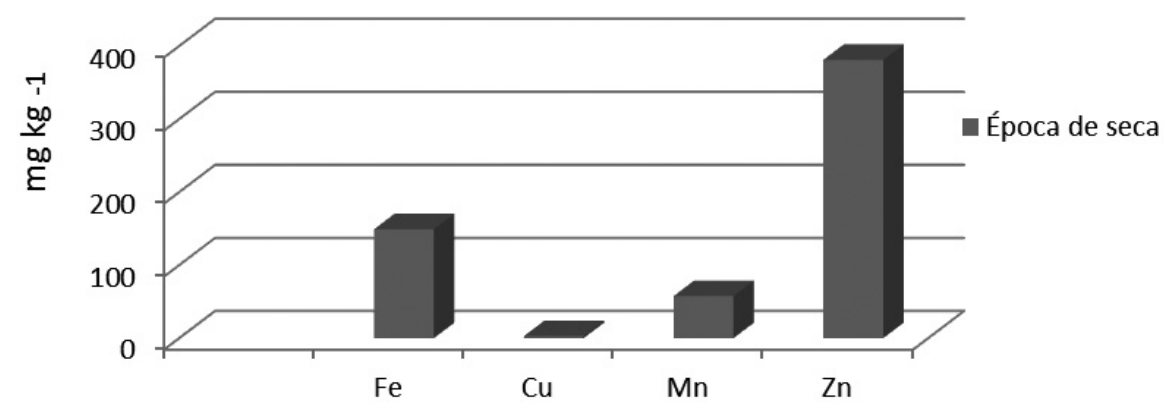

FIGURA 5- Concentración de los microelementos en área foliar de guanábano en época seca.

\section{Árbol no productivo}

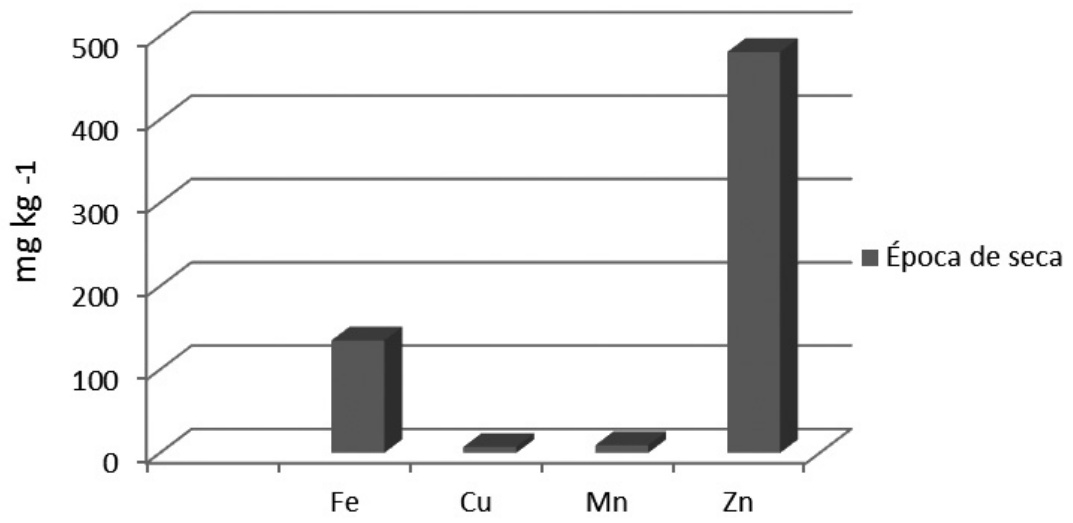

FIGURA 6- Concentración de los microelementos en área foliar de guanábano en época seca.

\section{Contenido de macroelementos (\%)}

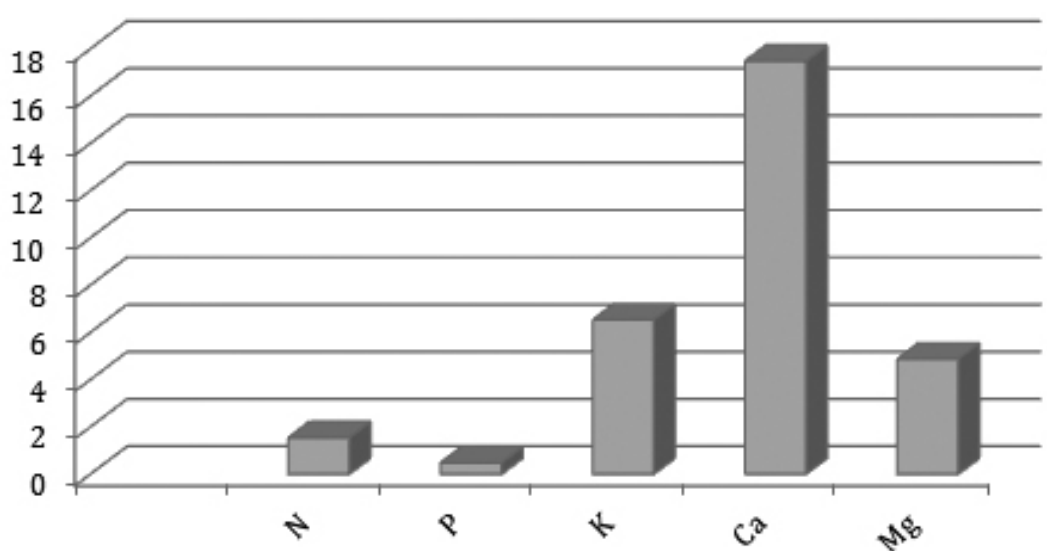

FIGURA 7- Contenido de macroelementos en muestras de suelo durante la época de lluvia. 
Contenido de microelementos $\left(\mathrm{mg} \mathrm{kg}^{-1}\right)$

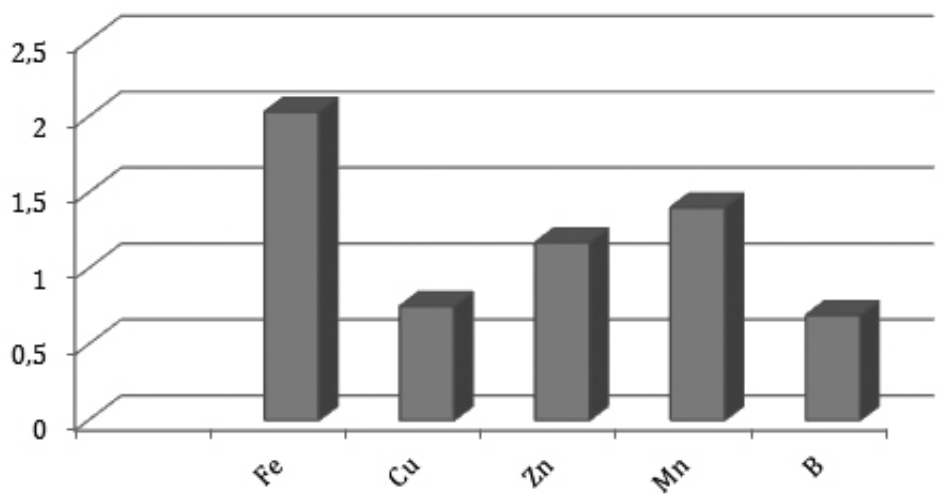

FIGURA 8- Contenido de microelementos en muestras de suelo durante la época de lluvia

\section{Contenido de microelementos $\left(\mathrm{mg} \mathrm{kg}^{-1}\right)$}

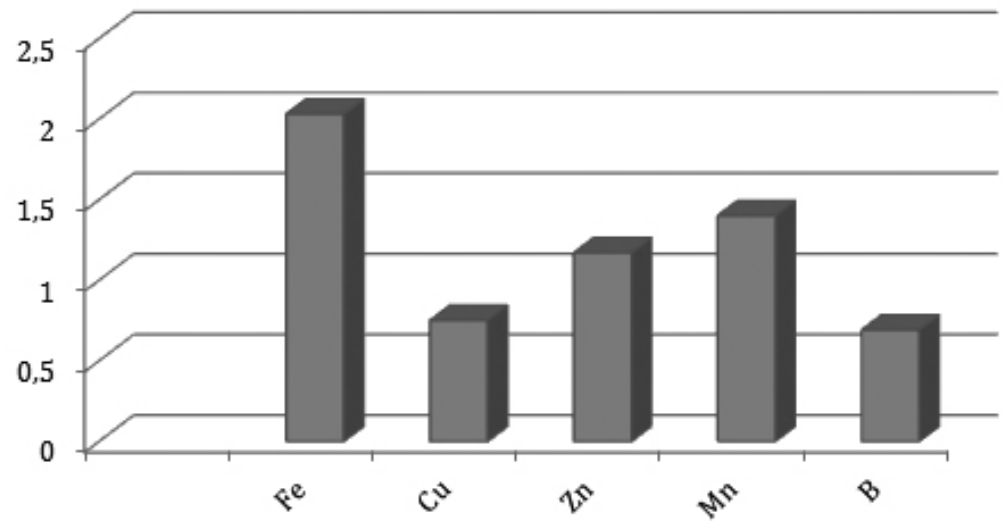

FIGURA 9- Concentración de macroelementos en muestras foliar durante la época de lluvia.

\section{Árbol no productivo}

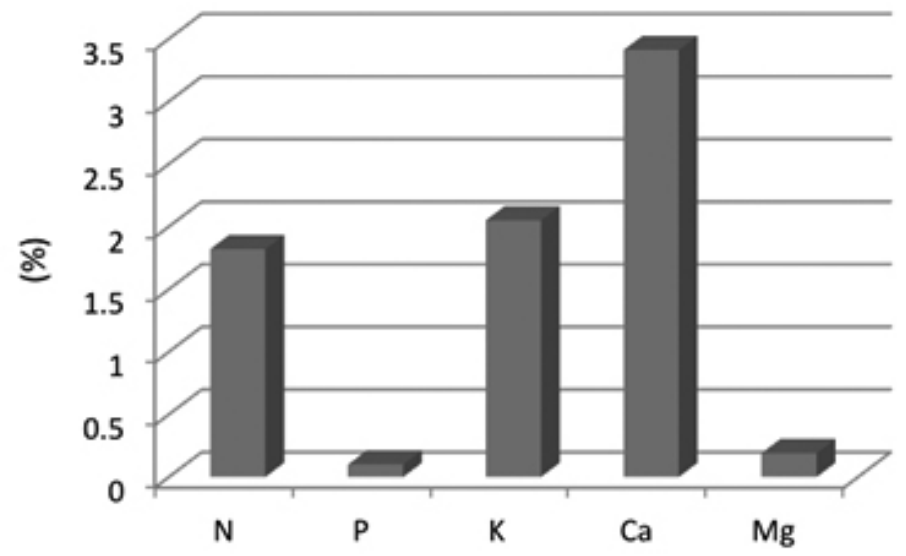

FIGURA 10- Concentración de macroelementos en muestras foliar durante la época de lluvia. 


\section{Árbol productivo}

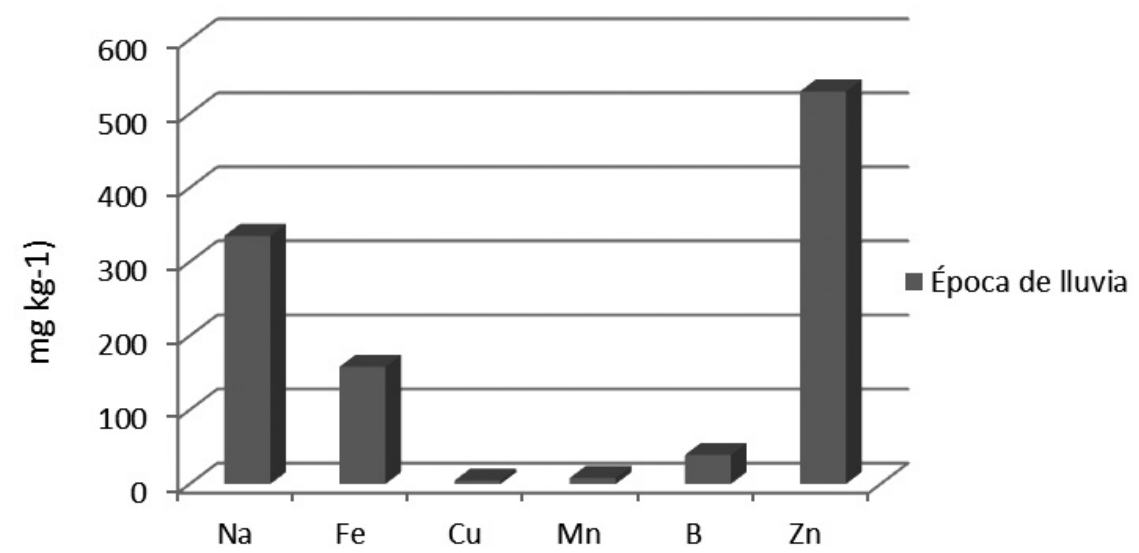

FIGURA 11- Concentración de microelementos en muestras foliar durante la época de lluvia.

\section{Árbol no productivo}

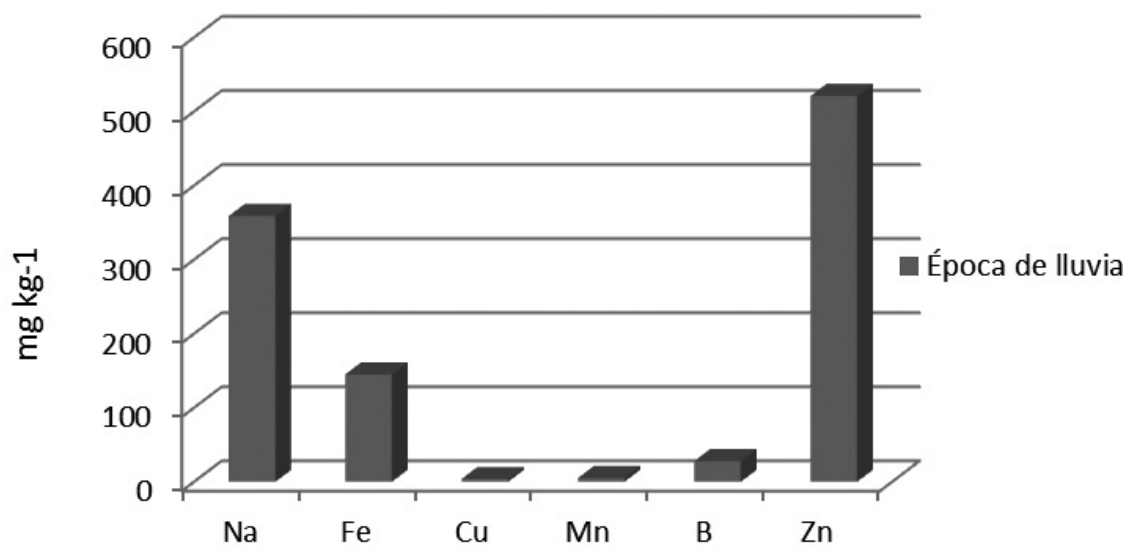

FIGURA 12-Concentración de microelementos en muestras foliar durante la época de lluvia.

\section{REFERENCIAS}

ÁLVAREZ, G.F. Evaluación agronómica de frutales en once comunidades de Centla, Tabasco; bajo la influencia de lluvia ácida. 1996. $106 \mathrm{f}$. Tesis (Profesional) - Facultad de Ciencias Agrícolas, Campus Xalapa, Universidad Veracruzana, 1996.

AVILÁN L. R. Efecto de la omisión de los macronutrientes em el desarrollo y composición química de la guanábana (Annona muricata L.) cultivada en soluciones nutritivas.. Agronomía Tropical , Maracay, v.25, n.1, p. 73-79, 1975.
CASTRO F. H. E. Base técnica para el conocimiento y manejo del suelo del Valle del Alto Magdalena. Palmira: Corpoica, 1996. 109 p.

ELIZONDOM. R. Consideraciones agroeconómicas del guanábano (Annona muricata L.), en Costa Rica. San José: MAG, 1989. 207 p.

GONZÁLEZ A. L. Diagnóstico nutrimental y patológico del cultivo de guanábana (Annona muricata L.) en el estado de Tabasco. 2003. 117 f. Tesis (Maestría) - Colegio de Postgraduados H. Cárdenas, Tabasco, 2003. 
MALDONADO T. R., VERGARA, S. M. A. Diagnóstico nutrimental de naranja (Citrus sinensis L. Osbeck) en varias regiones citrícolas de México. In: SISTEMAS DE PRODUCCIÓN EN CÍTRICOS, 2., 1993, Chapingo. Memorias... Chapingo: Universidad Autónoma, 1993. p.111-121.

SEMARNAT - Secretaria del Medio Ambiente y Recursos Naturales. Que establece las especificaciones de fertilidad, salinidad y clasificación de suelos. Estudios, muestreo y análisis. Non-021Semarnat-2000. Diario Oficial, México, 31 dic. 2002.
VERGARA S. M. A. Análisis foliar y su interpretación (DRIS). Chapingo: Universidad Autónoma Chapingo, 1990.

VIDAL H. L. La Guanábana. Tuxpan: Facultad de Ciencias Agrícolas, Zona. Universidad Veracruzana, 1993. 16 p. (Publicación Técnica, 2)

VIDAL H. L. Fitotecnia del guanábano. In: CURSO DE ACTUALIZACIÓN FRUTÍCOLA, 19. 2006, Temascaltepec. Memoria... Temascaltepec: Fundación Salvador Sánchez Colín, 2006. p.65-81 